\title{
Pushing the detection limits: The evanescent field in surface plasmon resonance and analyte-induced folding observation of long human telomeric repeats
}

\author{
Constanze Schlachter ${ }^{\mathrm{a}, \mathrm{b}}$, Fred Lisdat ${ }^{\mathrm{b}, *}$, Marcus Frohme $^{\mathrm{b}}$, Volker A. Erdmann ${ }^{\mathrm{c}}$, Zoltán Konthur ${ }^{\mathrm{a}}$, \\ Hans Lehrach ${ }^{\mathrm{a}}$, Jörn Glökler ${ }^{\mathrm{a}, \mathrm{d}, * *}$ \\ a Department of Vertebrate Genomics, Max Planck Institute for Molecular Genetics, Ihnestrasse 63-73, 14195 Berlin, Germany \\ b Biosystems Technology, Technical University of Applied Sciences, Bahnhofstraße, 15745 Wildau, Germany \\ ' Institute for Chemistry/Biochemistry, Free University Berlin, Thielallee 63, 14195 Berlin, Germany \\ d Alacris Theranostics GmbH, Fabeckstr. 60, 14195 Berlin, Germany
}

\section{A R T I C L E I N F O}

\section{Article history:}

Received 6 September 2011

Received in revised form 2 November 2011

Accepted 3 November 2011

Available online 10 November 2011

\section{Keywords:}

Evanescent field

Surface plasmon resonance

Long concatemeric repeat

Human telomere

Ligand-induced folding

\begin{abstract}
A B S T R A C T
Conventional analysis of molecular interactions by surface plasmon resonance is achieved by the observation of optical density changes due to analyte binding to the ligand on the surface. Low molecular weight interaction partners are normally not detected. However, if a macromolecule such as DNA can extend beyond the evanescent field and analyte interaction results in a large-scale contraction, then the refractive index changes due to the increasing amount of macromolecules close to the surface. In our proof-ofprinciple experiment we could observe the direct folding of long, human telomeric repeats induced by the small analyte potassium using surface plasmon resonance spectroscopy. This work demonstrates the feasibility of new evanescent field-based biosensors that can specifically observe small molecule interactions.
\end{abstract}

(C) 2011 Elsevier B.V. All rights reserved.

\section{Introduction}

The impact of surface plasmon resonance for the analysis of molecular interactions has been tremendous since its introduction in 1983 (Liedberg et al., 1983). This becomes obvious by the amount of publications growing almost exponentially each year (Knoll et al., 2008). Some of the most straightforward SPR experiments involve nucleic acid interactions, such as hybridization and protein binding, typical for nucleic acid biochemistry. However, in addition to encoding the genetic information, nucleic acids are known to form complex secondary structures different from double helices, such as tRNAs that can fold in a certain structure important for its biological function. A well known three-dimensional structure of nucleic acids is the G-quadruplex which is formed in vitro by DNA and RNA sequences occurring in telomeres, promoter regions, recombination sites, RNA packaging sites and RNA dimerization domains (Shafer and Smirnov, 2000; Arthanari and

\footnotetext{
* Corresponding author at: Department of Biosystems Technology, Technical University of Applied Sciences, Bahnhofstraße, 15745 Wildau, Germany. Tel : +49 03375 508456x799; fax: +49 03375508578

** Corresponding author at: Department of Vertebrate Genomics, Max Planck Institute for Molecular Genetics, Ihnestrasse 63-73, 14195 Berlin, Germany.

E-mail addresses: flisdat@th-wildau.de (F. Lisdat), j.gloekler@alacris.de, gloekler@molgen.mpg.de (J. Glökler).
}

Bolton, 2001; Simonsson, 2001), or artificially generated aptamers (Griffin et al., 1993; Gatto et al., 2009). In 2009 Blackburn, Greider and Szostak were awarded the Nobel Prize in Medicine for the discovery of the mechanism of telomeric protection of chromosomes and the enzyme telomerase (Szostak and Blackburn, 1982; Greider and Blackburn, 1985, 1989). Telomeres, i.e. the termini of the chromosomes, consist of a $d$ (GGGTTA $)_{n}$ repeat sequence, which is the same in all vertebrate genomes. Human telomeres measure between 5 and 15 kilobases (Samassekou et al., 2010), of which the 3 '-end of the G-rich strand forms an overhang, measuring between 35 and 600 nucleotides (Makarov et al., 1997; McElligott and Wellinger, 1997; Stewart et al., 2003). Besides protecting the end of chromosomes, it is shown that the overhang of telomeres can fold into G-quadruplexes in vivo (Blackburn, 1991). In these Grich sequences guanine bases interact via Hoogsteen base pairing to form a three-dimensional G-quadruplex structure. The folding is supported by binding to monovalent cations. The binding is mainly dependent on the ion radius with a strong preference for potassium over other cations, especially under physiologically relevant concentrations (Hardin et al., 1991; Renciuk et al., 2009).

The structural change can be measured directly by e.g. fluorescence resonance energy transfer (FRET) with the disadvantage of using labels (Simonsson and Sjöback, 1999) or direct probing by atomic force microscopy (Basnar et al., 2006).

Using the principle of surface plasmon (SPR) resonance, a direct and on-line measurement without the need of labels can be 
A

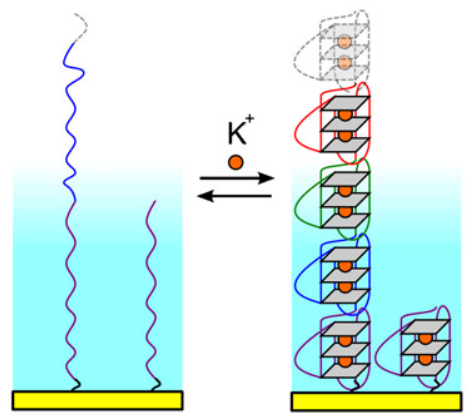

B

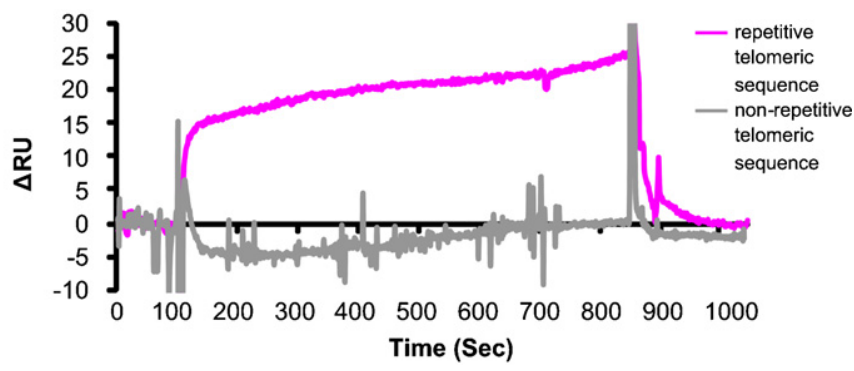

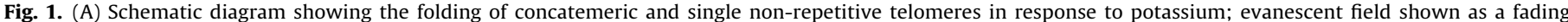

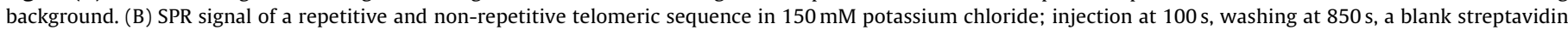
coated surface was used as a reference for normalization.

performed. With SPR it is possible to detect changes in the refractive index as a result of binding events or changes of the structure of biomolecules, such as RNA (Lisdat et al., 2001; Willander and Al-Hilli, 2009). However, structural changes of one G-quadruplex forming sequence were not detectable with this method so far (Redman, 2007). Until now, only indirect measurements of the folding of a telomeric sequence have been applied (Zhao et al., 2004). Since long telomeric repeats forming G-quadruplexes occur naturally, these can be ideally synthesized in vitro by rolling circle amplification (RCA) (Pomerantz et al., 2008). In this process, a circular template is elongated by the highly processive $\varphi 29$ DNA polymerase to form extremely long single-stranded concatemeric repeats mainly depending on the incubation time (Banér et al., 1998). Here, we present the proof-of-principle of combining the advantages of SPR with the potential of RCA for direct measurement of the structural changes of a repetitive human telomeric motif in the presence of potassium ions. We intend to capitalize on the limitations of SPR represented by the extent of the evanescent field allowing refractive index change measurements only close to the surface. Experiments conducted by Lavine et al. (2007) using the differential swelling behavior of molecularly imprinted polymers in SPR have shown that this can be done in principle. Therefore, very long single-stranded nucleic acids with the repetitive quadruplexforming motif and oligonucleotides with a repetitive scrambled telomeric sequence are synthesized by RCA from circularized templates. The scrambled telomeric sequence is used as a control, for another comparison an oligonucleotide with only one telomeric motif is applied (Fig. 1A). More detailed information about materials and methods used is provided in the Supporting Information.

\section{Results and discussion}

To evaluate the advantage of a repetitive telomeric sequence over a sequence with one telomeric motif, we first observe the SPR signal in the presence of $150 \mathrm{mM}$ potassium chloride for single and long telomeric repeats (Fig. 1B).

As expected, it is not possible to detect the G-quadruplex folding induced by potassium ions directly in case of single non-repetitive telomeric sequences. On the other hand, we find an increasing SPR signal for the repetitive telomeric sequence after contact with the potassium containing buffer. This shift in signal is possibly caused by the formation of Gquadruplexes in the presence of potassium ions. For further studies we use isomolar buffers of $150 \mathrm{mM}$ monovalent ions with varying concentrations of potassium to lithium (Fig. 2). This setup is chosen to prevent large bulk index shifts that overlay the test signal. DNA strands with the scrambled telomeric sequence are used as a negative control, as they cannot form G-quadruplex structures (Pomerantz et al., 2008). As can be seen from Fig. 2B, no change in the refractive index can be detected, in contrast to the repetitive G-quadruplex forming DNA. Thus, the SPR signal change for the latter can be attributed to a conformational change of this DNA-layer.

Besides the occurrence of conformational changes, it is also possible to observe the kinetics of the folding process since the signal increases with injection time (Fig. 2A). The response curves for various potassium concentrations indicate a fast response followed by a slower process. Obviously, repetitive structures are not able to fold in a single step because folding of such large molecules is sterically challenging and is combined with contraction. Repetitive scrambled strands show no specific signal in the presence of various potassium concentrations. In contrast, repetitive telomeric strands reveal a signal, which is dependent on the potassium ion concentration (Fig. 2B). Changes in
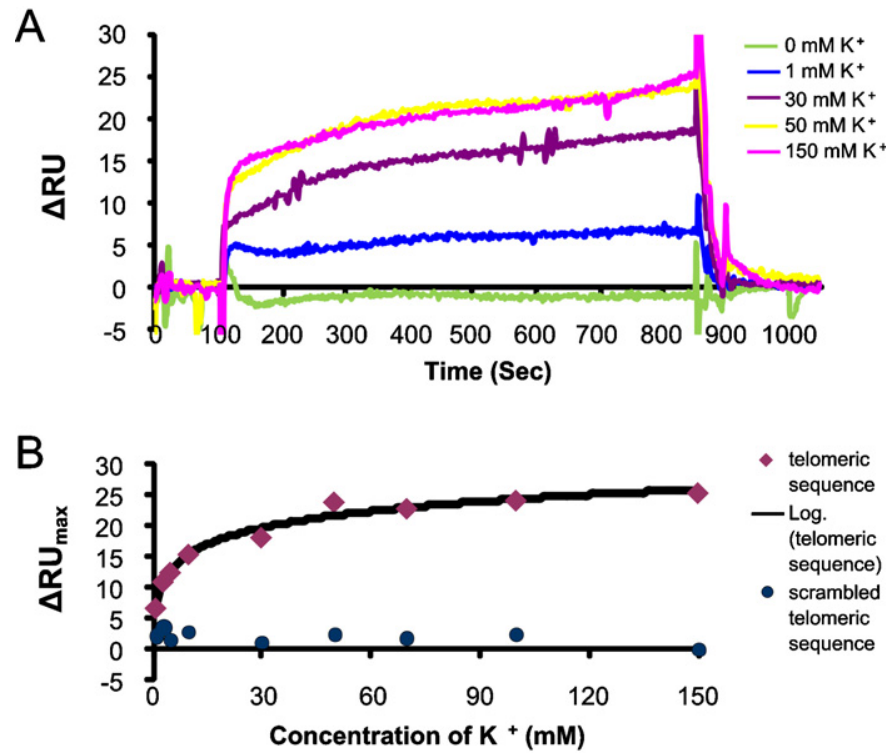

Fig. 2. Concentration dependence of the SPR signal on different potassium concentrations. (A) Representative sensorgrams of the repetitive telomeric sequence for potassium concentrations from 0 to $150 \mathrm{mM}$; injection at $100 \mathrm{~s}$, washing at $850 \mathrm{~s}$, a blank streptavidin surface was used as a reference for normalization. (B) Maximal association signal for the different concentrations in comparison of repetitive scrambled telomeric strand (blue dots) and repetitive telomeric strand (purple diamonds) showing a logarithmic dependence of the potassium concentration for the telomeric sequence. 
A

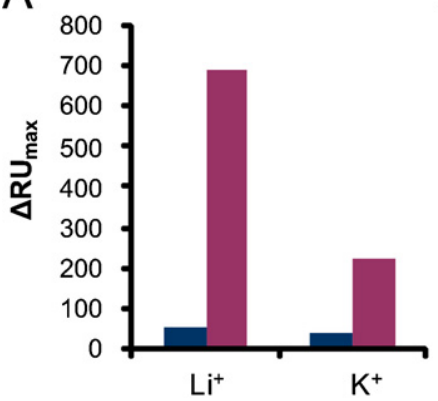

B

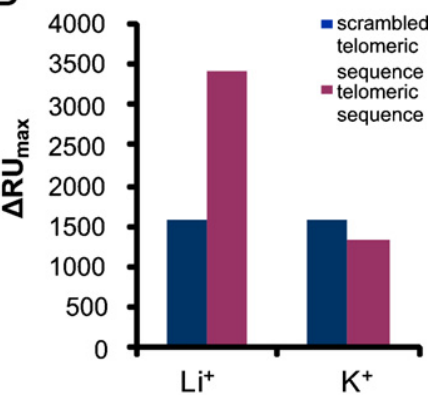

Fig. 3. Binding signals of complementary strands (A) or SSB (B) to long repetitive telomeric and long repetitive scrambled telomeric sequences in the absence or presence of $150 \mathrm{mM} \mathrm{K}^{+}$ions and $\mathrm{Li}^{+}$ions. (A) Results of the injection of $500 \mathrm{nM} \mathrm{com-}$ plementary scrambled oligonucleotide to the scrambled telomeric sequence and $500 \mathrm{nM}$ of the complementary telomeric oligonucleotide to the telomeric sequence. (B) Binding signals of the injection of 150 nM SSB.

the refractive index already occur at $1 \mathrm{mM}$ concentration. These results indicate a direct measurement of the complex folding of a G-quadruplex structure with surface plasmon resonance spectroscopy.

In order to confirm the specificity of the signal for G-quadruplex folding an indirect measurement for the detection of a quadruplex structure is performed. We use hybridization of complementary sequences to determine the presence of a G-quadruplex structure. In case of G-quadruplex formation, the complementary strand is not able to bind because the sequence is already involved in this secondary structure. However, hybridization is possible if the strand is present in its linear form. In addition to complementary oligonucleotides, we use the single-strand binding protein (SSB) from Escherichia coli to probe the secondary structure. The obtained binding signals in the absence or presence of potassium ions are given in Fig. 3.

The indirect measurements confirm that G-quadruplex formation in the telomeric sequence takes place in the presence of potassium ions, since binding of the complementary strand decreases. In the absence of potassium and presence of lithium, no secondary structure is formed allowing the complementary strand to hybridize. At the same time, the scrambled telomeric sequence exhibit almost the identical binding signal for the specific complementary oligonucleotide, both, in the absence or presence of potassium, confirming the absence of any $G$ quadruplex structures. The explanation for the low binding of the scrambled sequence lies partially in a slight difference of the total amount of immobilized DNA (see Supplementary data). Additionally, the scrambled sequence can form more stable secondary structures than the telomere. We have checked the scrambled sequence for hairpin formation by UNAFold software (Markham and Zuker, 2008) and find one hairpin that forms at $27^{\circ} \mathrm{C}$ and a second at $22^{\circ} \mathrm{C}$. In contrast, the telomeric sequence forms only one hairpin at $22^{\circ} \mathrm{C}$. This difference is amplified by the additional strong hairpin formation of the scrambled antisense oligonucleotide used for the hybridization test $\left(40.3^{\circ} \mathrm{C}\right)$, whereas the telomeric antisense oligonucleotide remains unfolded.

Similarly, binding experiments with SSB to both kinds of sequences show that the signal is decreased in the presence of potassium for the telomeric sequence and equivalent, both, in the absence or presence of potassium for the scrambled telomeric sequence. The different binding abilities of SSB to the two sequences in the $\mathrm{Li}^{+}$-containing buffer may result from not identical structures of the two different DNA layers as described above without the additional effect of an antisense structure.
In order to demonstrate the reliability of the measurements, the same hybridization and SSB experiments are conducted with short, non-repetitive sequences. They reveal that the nonrepetitive telomeric sequence also forms G-quadruplex structures in potassium solution (see Supplementary data) and thus can neither hybridize to complementary sequences, nor bind SSB. Note, that this change in conformation is not possible to be observed directly, but can only be confirmed by these two indirect measurements.

In conclusion, this study represents the first direct measurement of structural changes, such as G-quadruplex formation, induced by small molecules, such as potassium ions, with SPR. The direct and label-free detection has been achieved by the use of long nucleic acid molecules with repetitive units. Due to this repetitive order of the quadruplex-forming sequence, an amplification of the signal occurs enabling direct analysis. Measurements with different concentrations of potassium ions clearly show a concentration dependent folding process. The formation of the G-quadruplex structure in the experiments is confirmed by a decreased binding capacity for complementary sequences or SSB. Various control sequences give no effect under the same experimental conditions. Therefore, the obtained signals are specific for the structural transition of the long, telomeric repeats from a disordered conformation into G-quadruplexes.

The most likely explanation for this phenomenon is the extreme length of the concatemeric repeats generated by RCA that extend beyond the evanescent field measured some hundred $\mathrm{nm}$ from the surface (Knoll, 1998; Van Der Merve, 2001). Applying RCA, more than 50 kilobases can be polymerized per hour (Basnar et al., 2006) and correspondingly lengths exceeding $4 \mu \mathrm{m}$ have been observed under the microscope (Pomerantz et al., 2008). Upon contraction of the telomeric repeats, more units are reeled in and thus increase the refractive index.

In the past many other techniques e.g. optodes, have been used to measure potassium (Krause et al., 1999). However, the dynamic range of such measurements is in the $5-100 \mathrm{mM}$ range. Especially if physiological concentrations of extracellular potassium of 3.5-6 mM (Katzman, 1976) are to be measured, our quadruplex biosensor approach may be advantageous. A certain fine-tuning of the dynamic range with respect to melting temperature, buffer composition, and potassium sensitivity can be achieved by systematic mutagenesis of known quadruplex sequences (Guédin et al., 2010).

However, this study is focused on a new detection principle and thus implies the ability of direct measurements of structural changes of DNA due to binding of low molecular weight analytes, such as potassium ions, with SPR in general. One field of application can be seen in aptamer structures which fold in the presence of an analyte. When the analyte is small, the conformational change of the repetitive recognition layer might be easily detected. Several evanescent field biosensor principles could be adapted in a similar manner (Taitt et al., 2005). Especially nanoplasmonic sensors with evanescent fields that decay more rapidly have been shown to respond to structural changes of bulky molecules (Jonsson et al., 2007, 2008). These could be combined with much shorter concatemeric repeats than those used in our study. Alternatively, sensors that more directly measure the macroscopic effect of mechanical contraction may become feasible using very long concatemeric repeats. A further interesting application without measuring any ligand binding is to detect DNA damages that inhibit structure formation (Esposito et al., 2010). Additionally, this newly discovered principle may not be limited to covalently linked concatemeric repeats, but also be applied to higher order structures assembled by techniques such as DNA origami (Seeman, 2003; Majumder et al., 2011). 


\section{Acknowledgements}

This work was partially supported by the European Union through the EFRE program [ProFIT grant no. 10139409] and the BmBF [grant no. 13N9593].

\section{Appendix A. Supplementary data}

Supplementary data associated with this article can be found, in the online version, at doi:10.1016/j.bios.2011.11.003.

\section{References}

Arthanari, H., Bolton, P.H., 2001. Chem. Biol. 8, 221-230.

Banér, J., Nilsson, M., Mendel-Hartvig, M., Landegren, U., 1998. Nucleic Acids Res. 26, 5073-5078.

Basnar, B., Elnathan, R., Willner, I., 2006. Anal. Chim. 78, 3638-3642.

Blackburn, E.H., 1991. Nature 350, 569-573.

Esposito, V., Martino, L., Citarella, G., Virgilio, A., Mayol, L., Giancola, C., Galeone, A., 2010. Nucleic Acids Res. 38, 2069-2080.

Gatto, B., Palumbo, M., Sissi, C., 2009. Curr. Med. Chem. 16, 1248-1265.

Greider, C.W., Blackburn, E.H., 1985. Cell 43, 405-413.

Greider, C.W., Blackburn, E.H., 1989. Nature 337, 331-337.

Griffin, L.C., Tidmarsh, G.F., Bock, L.C., Toole, J.J., Leung, L.L., 1993. Blood 81, 3271-3276.

Guédin, A., Gros, J., Alberti, P., Mergny, J.-L., 2010. Nucleic Acids Res. 38, 7858-7868. Hardin, C.C., Henderson, E., Watson, T., Prosser, J.K., 1991. Biochemistry 30, 4460-4472.

Jonsson, M.P., Jönsson, P., Dahlin, A.B., Höök, F., 2007. Nano Lett. 7, 3462-3468.

Jonsson, M.P., Jönsson, P., Höök, F., 2008. Anal. Chem. 80, 7988-7995.

Katzman, R., 1976. Fed. Proc. 35, 1244-1247.
Knoll, W., 1998. Annu. Rev. Phys. Chem. 49, 569-638.

Knoll, W., Kasry, A., Liu, J., Neumann, T., Niu, L., Park, H., Paulsen, H., Robelek, R. Yao, D., Yu, F., 2008. In: Schasfoort, R.B.M., Tudos, A.J. (Hrsg.), Handbook of Surface Plasmon Resonance. Royal Society of Chemistry, Cambridge, pp. 275-312 (Chapter 9).

Krause, C., Werner, T., Huber, C., Wolfbeis, O.S., 1999. Anal. Chem. 71, 5304-5308. Lavine, B.K., Westover, D.J., Kaval, N., Mirjankar, N., Oxenford, L., Mwangi, G.K., 2007. Talanta 72, 1042-1048.

Liedberg, B., Nylander, C., Lunström, I., 1983. Sens. Actuators 4, 299-304.

Lisdat, F., Utepbergenov, D., Haseloff, R.F., Blasig, I.E., Stöcklein, W., Scheller, F.W. Brigelius-Flohé, R., 2001. Anal. Chem. 73, 957-962

Majumder, U., Rangnekar, A., Gothelf, K.V., Reif, J.H., Labean, T.H., 2011. J. Am. Chem. Soc. $133,3843-3845$.

Makarov, V.L., Hirose, Y., Langmore, J.P., 1997. Cell 88, 657-666.

Markham, N.R., Zuker, M., 2008. Methods Mol. Biol. 453, 3-31.

McElligott, R., Wellinger, R.J., 1997. EMBO J. 16, 3705-3714.

Pomerantz, A.K., Moerner, W.E., Kool, E.T., 2008. J. Phys. Chem. B 112, 13184-13187.

Redman, J.E., 2007. Methods 43, 302-312.

Renciuk, D., Kejnovská, I., Skoláková, P., Bednárová, K., Motlová, J., Vorlícková, M. 2009. Nucleic Acids Res. 37, 6625-6634.

Samassekou, O., Gadji, M., Drouin, R., Yan, J., 2010. Ann. Anat. 192, 284-291.

Seeman, N.C., 2003. Nature 421, 427-431.

Shafer, R.H., Smirnov, I., 2000. Biopolymers 56, 209-227.

Simonsson, T., 2001. Biol. Chem. 382, 621-628.

Simonsson, T., Sjöback, R., 1999. J. Biol. Chem. 274, 17379-17383.

Stewart, S.A., Ben-Porath, I., Carey, V.J., O'Connor, B.F., Hahn, W.C., Weinberg, R.A. 2003. Nat. Genet. 33, 492-496.

Szostak, J.W., Blackburn, E.H., 1982. Cell 29, 245-255.

Taitt, C.R., Anderson, G.P., Ligler, F.S., 2005. Biosens. Bioelectron. 20, 2470-2487.

Van Der Merve, P.A., 2001. Protein-Ligand Interactions: A Practical Approach. Oxford University Press, pp. 1-50.

Willander, M., Al-Hilli, S., 2009. Methods Mol. Biol. 544, 201-229.

Zhao, Y., Kan, Z.Y., Zeng, Z.X., Hao, Y.H., Chen, H., Tan, Z., 2004. J. Am. Chem. Soc. 126 , $13255-13264$. 